\title{
Multiple origins of U genome in two UM genome tetraploid Aegilops species, Ae. columnaris and Ae. triaristata, revealed based on the polymorphism of a genome-specific PCR fragment
}

\author{
Sayo Kadosumi ${ }^{1}$, Taihachi Kawahara ${ }^{2}$ and Tsuneo Sasanuma ${ }^{1 *}$ \\ ${ }^{1}$ Division of Evolutionary Genetics, Kihara Institute for Biological Research, Yokohama City \\ University, Maioka-cho 641-12, Totsuka-ku, Yokohama 244-0813, Japan \\ ${ }^{2}$ Laboratory of Crop Evolution, Plant Germ-plasm Institute, Graduate School of \\ Agriculture, Kyoto University, Mozume, Muko, Kyoto 617-0001, Japan
}

(Received 4 January 2005, accepted 25 April 2005)

\begin{abstract}
To elucidate the evolutionary mode of the formation of species via polyploidization, we conducted phylogenetic analysis of the U genome of the UM genome tetraploid Aegilops species, Ae. columnaris and Ae. triaristata. Using the genomespecific PCR primer set U31, we investigated the variation of the U genome of 48 accessions each of Ae. columnaris and Ae. triaristata and 72 accessions of their diploid ancestor Ae. umbellulata. As a result, three alleles were distinguishable by amplified length and CAPS polymorphisms, namely, allele I = normal size with an $M s p$ I site, allele II = normal size without an $M s p$ I site, and allele III = shorter size caused by a $123 \mathrm{bp}$ deletion. All three alleles were detected both in diploid and tetraploid accessions. Sequence comparison indicated the inheritance of alleles I and III from the diploid to the tetraploids, suggesting multiple origins of the U genome of the tetraploids. Regarding allele II, however, the sequence comparison indicated that parallel mutations at the $M s p$ I site produced allele II several times. The phylogenetic tree based on the sequences of the U31 region demonstrated the presence of a third lineage of the U genome from Ae. umbellulata to Ae. columnaris. Consequently, we concluded that the $\mathrm{U}$ genome had at least three origins in Ae. columnaris, and at least two, probably more, in Ae. triaristata.
\end{abstract}

Key words: Ae. columnaris, Ae. triaristata, multiple origins, polyploidy, U genome-specific primer

\section{INTRODUCTION}

Polyploidization, the stable amplification or coexistence of more than two genomes in a nucleus, has played an important role in plant evolution. Masterson (1994) reported that $70 \%$ of angiosperms have experienced genome duplication at least once. Researchers have long pointed out the evolutionary potential of polyploidization, that is, it may produce more genetic diversity in nuclear genomes and consequently allow polyploids to be distributed in a wider area and to adapt to more varied niches than diploids (Kihara, 1954; Levin, 1980; Osborn et al., 2003).

The taxonomical complex of the genera Aegilops and Triticum (goatgrass and wheat, respectively) is one of the

Edited by Minoru Murata

* Corresponding author. E-mail: sasanuma@yokohama-cu.ac.jp most extensively investigated groups with respect to allopolyploidy. Extensive genome analysis with cytological techniques revealed the diploid ancestors of most of the polyploids in these genera (Lilienfeld, 1951). Subsequently, plasmon analysis with molecular techniques determined the cytoplasm type for each species (Wang et al., 1997). In a study of the plasmon analysis, Murai and Tsunewaki (1986) found that Ae. triuncialis L., a UC genome tetraploid Aegilops species, has two reciprocal maternal lineage within a species. This was the first molecular evidence for multiple origins of polyploid species in these genera, although the possibility had been discussed because a high level of morphological variation exists in hexaploid wheat (Dekaprelevich, 1961; Kuckock, 1964; MacKey, 1966). The development of DNA technology and accumulation of information have made it possible to generate genome-specific primers, which facilitate the tracing of the nuclear lineage in polyploids. To date, 
several studies using such genome-specific primers have proved the existence of multiple origins of polyploids in these genera (Chee et al., 1995; Talbert et al., 1998; Vanichanon et al., 2003; Caldwell et al., 2004).

Ae. columnaris Zhuk. and Ae. triaristata Willd., which belong to the section Polyeides in the genus Aegilops, are tetraploids possessing the UM genome [nomenclature follows Kihara and Tanaka (1970)]. Their diploid ancestors are Ae. umbellulata Zhuk. for the U genome and Ae. comosa Sibth. et Sm. for the M genome, and the cyto- plasm donor for both species is Ae. umbellulata (Lilienfeld, 1951; Wang et al., 1997). These two UM genome species are morphologically similar and genetically close to each other (Kimber and Feldman, 1987; Resta et al., 1996; Badaeva, 2002). Among the section Polyeides, multiple origins of the U genome have been demonstrated for two tetraploid species, Ae. triuncialis and Ae. biuncialis Vis. (Chee et al., 1995; Vanichanon et al., 2003). This fact raised the question whether the other Polyeides polyploids also have multiple origins of the $\mathrm{U}$ genome. In

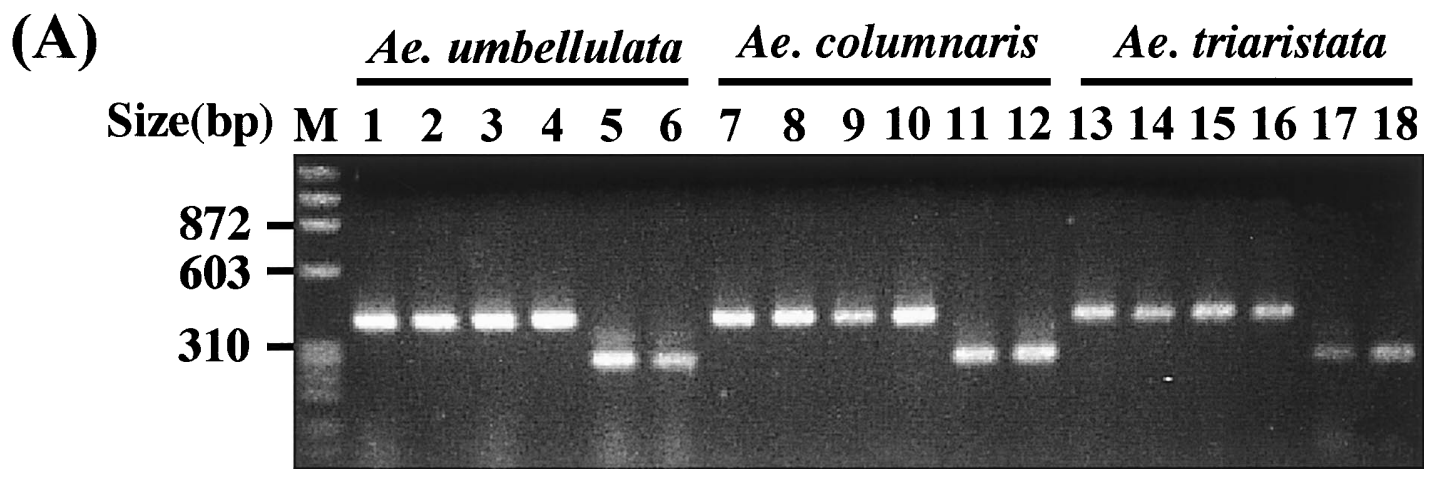

(B)

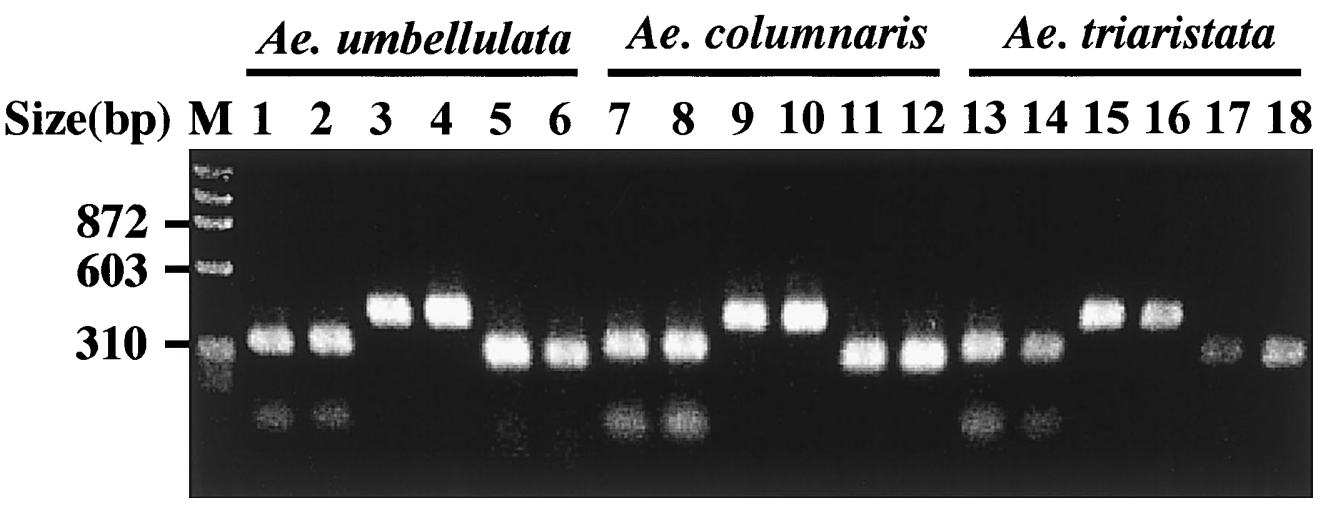

(C)
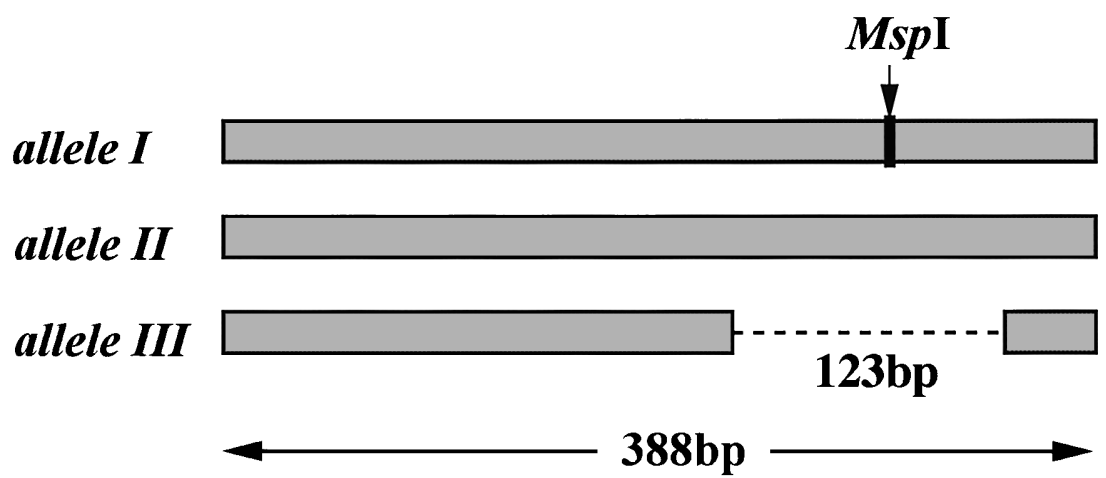

Fig. 1. Three alleles of locus U31. (A) Amplified length polymorphism of locus U31. Lanes 1-6 are Ae. umbellulata (accession code umb03, umb21, umb04, umb28, umb13, and umb14, from left to right), 7-12 Ae. columnaris (clm01, clm19, clm07, clm47, clm17 and clm22), and 13-18 Ae. triaristata (trr03, trr12, trr01, trr02, trr05 and trr48). M is size marker $\phi$ X174 digested with HaeIII. (B) CAPS profile of locus U31 digested with MspI. Sample order is the same as in (A). Lanes 1, 2, 7, 8, 13 and 14 represent allele I, 3, 4, 9, 10, 15 and 16 allele II, 5, 6, 11, 12, 17 and 18 allele III. (C) Schematic gene structure of three alleles of U31. The broken line indicates deletion. 
Table 1. Number of accessions for each allele of U31 in three Aegilops species

\begin{tabular}{llccc}
\hline \hline U31 Allele & Sequence at $M s p$ I site & Ae. umbellulata & Ae. columnaris & Ae. triaristata \\
\hline allele I & CCGG & $35(0.49)$ & $35(0.73)$ & $42(0.88)$ \\
allele II & & $27(0.38)$ & $2(0.04)$ & $4(0.08)$ \\
& CCAG & 21 & 1 & 0 \\
& CTGG & 3 & 0 & 4 \\
& CTGA & 3 & 0 & 0 \\
& CCG- & 0 & 1 & 0 \\
allele III & & $8(0.11)$ & $11(0.23)$ & $2(0.04)$ \\
allele IV (null) & & $2(0.03)$ & $0(0.00)$ & $0(0.00)$ \\
Total & & $72(1.00)$ & $48(1.00)$ & $48(1.00)$ \\
\hline
\end{tabular}

Frequency of each allele in the species is shown in the parenthesis. The number of accessions for each sequence type of allele II is shown in italics.

${ }^{\mathrm{a}}$ : - indicates a deletion.

Table 2. List of Aegilops accessions used for sequence analysis of entire U31 region

\begin{tabular}{|c|c|c|c|}
\hline Lab. code $^{a}$ & Accession No. $^{b}$ & Origin & U31 allele $^{\mathrm{c}}$ \\
\hline \multicolumn{4}{|c|}{ Ae. umbellulata } \\
\hline umb03 & KU2731 & Turkey & I \\
\hline umb21 & KU4109 & Iran & I \\
\hline umb32 & IG119380 & Syria & I \\
\hline umb04 & KU2760 & Turkey & II (CTGA) \\
\hline umb28 & IG49022 & Iraq & II (CCAG) \\
\hline umb31 & IG107074 & Iran & II (CCAG) \\
\hline umb13 & KU12189 & Greece & III \\
\hline umb14 & KU12199b & Greece & III \\
\hline umb25 & IG47866 & Turkey & III \\
\hline \multicolumn{4}{|c|}{ Ae. columnaris } \\
\hline $\operatorname{clm} 01$ & KU11-1 & Turkey & I \\
\hline $\operatorname{clm} 19$ & KU2953A & Armenia & I \\
\hline $\operatorname{clm} 24$ & KU5011 & Iraq & I \\
\hline $\operatorname{clm} 07$ & KU2706 & Iran & II (CCAG) \\
\hline $\operatorname{clm} 47$ & KU12779 & Syria & II (CCG-) \\
\hline $\operatorname{clm} 17$ & KU2719 & Iran & III \\
\hline $\operatorname{clm} 22$ & KU4194 & Turkey & III \\
\hline $\operatorname{clm} 43$ & KU6584 & Turkey & III \\
\hline \multicolumn{4}{|c|}{ Ae. triaristata } \\
\hline $\operatorname{trr} 03$ & KU2930 & Azerbaijan & I \\
\hline $\operatorname{trr} 12$ & KU4135 & Iraq & I \\
\hline $\operatorname{trr} 29$ & KU6244 & Turkey & I \\
\hline $\operatorname{trr} 01$ & KU10-4 & Turkey & II (CTGG) \\
\hline $\operatorname{trr} 02$ & KU10-3 & Turkey & II (CTGG) \\
\hline $\operatorname{trr} 37$ & KU6379 & Turkey & II (CTGG) \\
\hline $\operatorname{trr} 05$ & KU4122 & Iraq & III \\
\hline $\operatorname{trr} 48$ & KU4125 & Iraq & III \\
\hline \multicolumn{4}{|l|}{ Ae. bicornis } \\
\hline bcr01 & KU3-2 & Israel & II \\
\hline
\end{tabular}

${ }^{a}$ : Laboratory designated accession code used in this study.

${ }^{\mathrm{b}}$ : Accession number given by the original genebanks. KU and IG are the code indicating Kyoto University and ICARDA Genebank, respectively.

c: The polymorphic sequences at the MspI site are shown in the parentheses for allele II. - indicates a deletion. 
this study, we generated $\mathrm{U}$ genome-specific primers and applied them to large numbers of Ae. columnaris and Ae. triaristata, and the U-genome diploid Ae. umbellulata. The observed polymorphisms of the U genome-specific fragment revealed the multiplicity of the origin of the $U$ genome in these two species.

\section{MATERIALS AND METHODS}

Plant materials and DNA extraction A total of 72 accessions of Ae. umbellulata (accession code umb01 umb72) and 48 accessions each of Ae. columnaris (clm01 - clm48) and Ae. triaristata (trr01 - trr48) were used in this study. Thirteen accessions of Ae. umbellulata were provided by the genebank of the International Center for Agricultural Research in the Dry Areas (ICARDA) and all the rest were from the collection of the Plant Germ-plasm Institute, Graduate School of Agriculture, Kyoto University. Since Ae triaristata consists of tetraploid and hexaploid accessions, only tetraploid accessions were used in this study. The materials were chosen to cover most of the geographical distribution for each species. Total DNA was extracted from seedlings using a modified CTAB method (Murray and Thompson, 1980).

Construction of the $U$ genome-specific primers Several U genome-specific AFLP fragments detected previously (Sasanuma et al. 2004) were recovered from acrylamide gels and sequenced. Specific primers for each fragment were designed. The genome-specificity of the primers was verified by diagnostic PCR using all 12 diploid species of the genera Aegilops and Triticum. From the 39 AFLP fragments sequenced, one U genome-specific primer set, U31, was obtained. This primer set was used for the following analyses. This primer set amplified the $\mathrm{S}^{\mathrm{b}}$ genome of Ae. bicornis in addition to the $\mathrm{U}$ genome. The U31 region of the $\mathrm{S}^{\mathrm{b}}$ genome was included in the evolutionary analysis as an outgroup.

PCR amplification and CAPS analysis PCR was con-

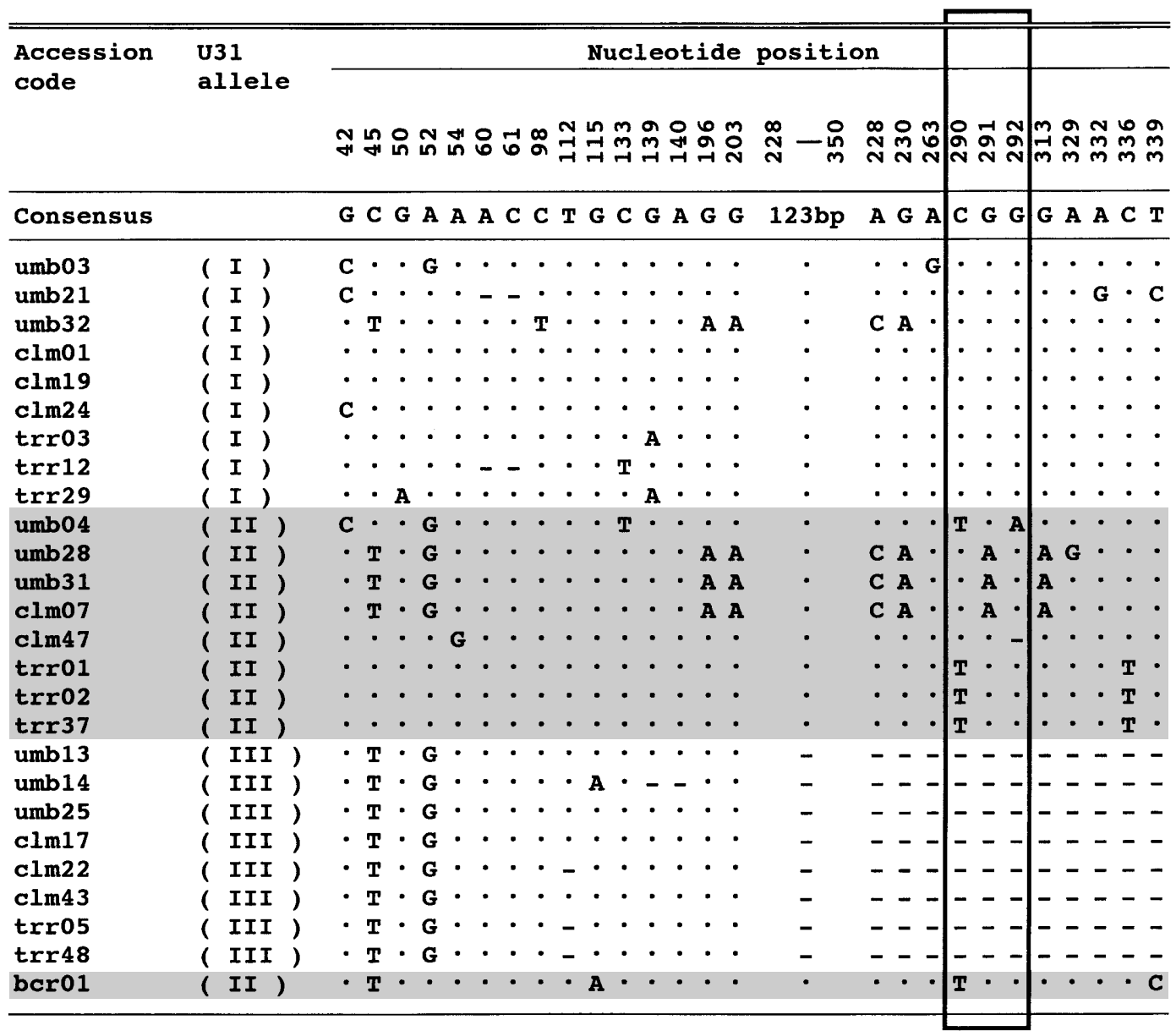

Fig. 2. Nucleotide substitutions detected in the U31 region among the 26 sequences. The nucleotides identical to the consensus sequence are represented by dots. Dashes mean deletions. The substitutions at the MspI site are highlighted by a bold-line square. The allele II sequences are shown by gray background. The 20 single substitutions detected only in bcr01 are not shown. 
ducted with the primer set U31 (U31a 5'-TATGATAAACAAGGCGAAAA-3' and U31b 5'-GGCTGTGGTGTATGATGACT-3'). The reaction conditions were 1 cycle of $94^{\circ} \mathrm{C}, 5 \mathrm{~min} ; 30$ cycles of $94^{\circ} \mathrm{C}, 1 \mathrm{~min} ; 55^{\circ} \mathrm{C}, 1 \mathrm{~min}, 72^{\circ} \mathrm{C}$, $2 \mathrm{~min}$; and finally 1 cycle of $72^{\circ} \mathrm{C}, 7 \mathrm{~min}$. PCR amplification of polymorphisms was investigated by electrophoresis in agarose gels. The U31 product of one accession each of the three species was sequenced to find restriction sites suitable for the cleaved amplified polymorphic sequence (CAPS) analysis. MspI restriction variation was thereby detected among the sequences. The amplified products of all accessions were accordingly digested with $M s p I$ and electrophoresed in agarose gels to examine the restriction polymorphism.

DNA sequencing The PCR products were sequenced for phylogenetic analysis. The infrared fluorescent IRD41-labeled U31a and U31b primers were used for sequencing with a DNA sequencer model 4000L (Aloka). To avoid errors of the polymerase reaction, the PCR products were directly used as the sequencing template. For construction of a phylogenetic tree, PAUP 3.1.1 (Swofford, 1993) was used.

\section{RESULTS}

PCR amplification and CAPS analysis of the U31 region Amplification length polymorphism was detected with PCR primer set U31 (Fig. 1A). Most of the accessions produced fragments with the expected size (approximately 390bp), but several accessions showed a product with shorter size (approx. 270bp). As shown in Fig. 1A, the shorter products were detected in all three species. In addition to these two types, we detected a non-amplified type in two accessions of Ae. umbellulata.

CAPS analysis with $M s p$ I revealed restriction polymorphism within a species (Fig. 1B). The products with normal size segregated into cleaved and non-cleaved types, while all the shorter-size products were non-cleaved. Consequently, based on both the amplification length and the restriction polymorphisms, the following three alleles were distinguishable; allele $\mathrm{I}=$ normal size with an $M s p \mathrm{I}$ site, allele II = normal size without an $M s p I$ site, and allele III $=$ shorter size. The structures of the three alleles are schematized in Fig. 1C. The non-amplified type was defined as allele IV. The number and frequency of the alleles in each species are summarized in Table 1. The three alleles, I, II and III, were present in all three species, although the frequency differed with the ploidy level. For all three species, the frequency of allele III was low.

Sequence analysis of the U31 region To verify the evolutionary orthology of each allele, we sequenced the $M s p I$ site and the deletion region of $\mathrm{U} 31$ for several accessions. In allele I, all 18 sequences determined from both the diploid (12 sequences) and the tetraploid accessions (three sequences for each) had an MspI site (CCGG) at the same position. Similarly, eight sequences of allele III from both the diploid (three sequences) and the tetraploids (three from Ae. columnaris and two from Ae. triaristata) contained a $123 \mathrm{bp}$ large deletion at the same position.

In contrast to these two alleles, allele II had variable nucleotide substitutions at the MspI site. Among all of the 33 accessions with allele II, four sequence types, CCAG, CTGG, CTGA and CCG-, were found at the MspI site. CCAG, CTGG and CTGA were detected in Ae. umbellulata, CCAG and CCG- in Ae. columnaris, and CTGG in Ae. triaristata. Table 1 shows the number of accessions for each sequence type. The outgroup species, Ae. bicornis, had allele II whose MspI site was CTGG.

Phylogenetic tree To reveal the evolutionary relationships among the alleles and species, we determined the

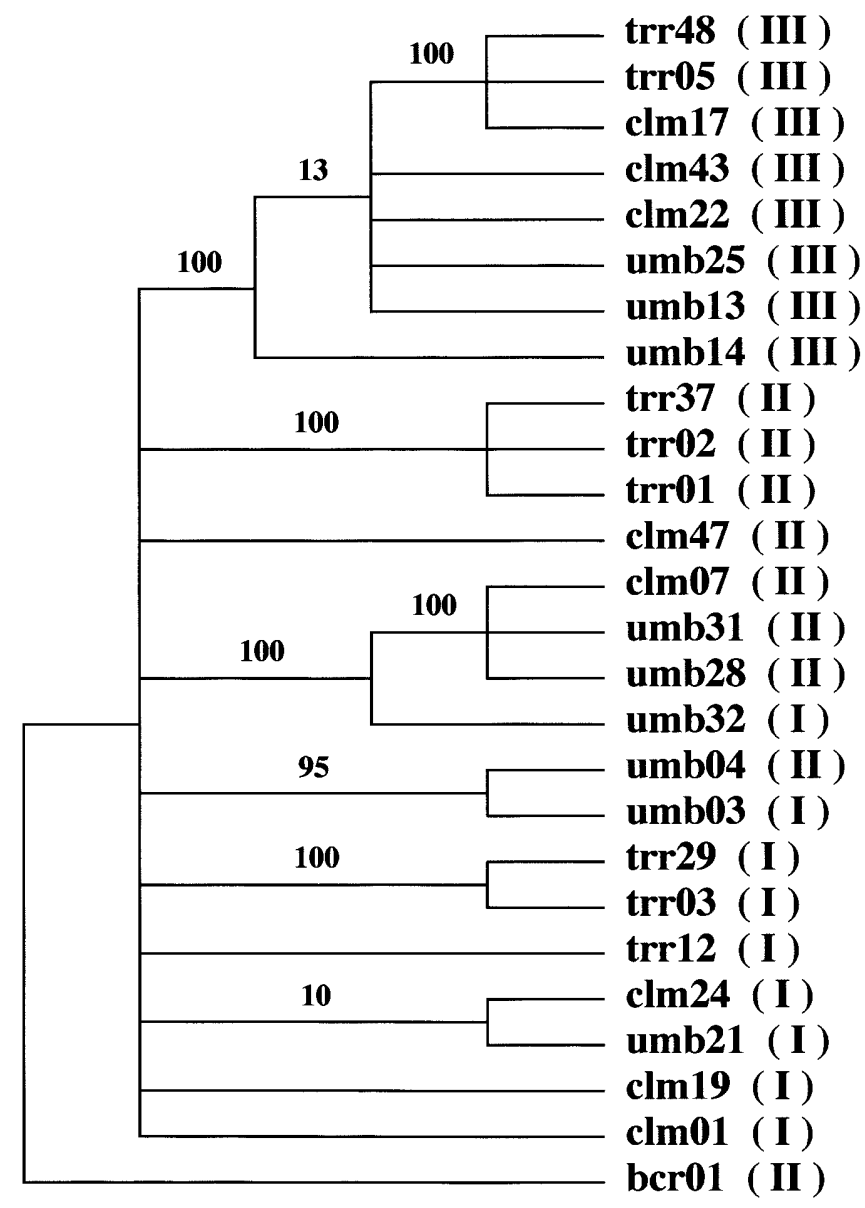

Fig. 3. Strict consensus tree of 39 minimal length trees for the U31 region. The length of the shortest trees was 180 . Numbers on the branches indicate the consensus indices for each cluster. Gaps were treated as a fifth base. The tree was rooted by defining the sequence of bcr01 as an outgroup. 
entire sequence of the U31 region for nine accessions of Ae. umbellulata and eight accessions each of Ae. columnaris and Ae. triaristata. The accessions used for sequencing are listed in Table 2. Excluding the primer sites and their flanking sequences, the alignment length was 339bp. Fig. 2 summarizes the nucleotide substitutions and Fig. 3 shows a strict consensus tree. In the tree, all eight sequences of allele III formed a distinct cluster. On the other hand, the sequences of alleles I and II were mixed with each other and dispersed in the tree showing a star-like topology. In detail, however, the three sequences of allele II with CCAG from Ae. umbellulata and Ae. columnaris (umb28, umb31 and clm07) formed a cluster. We could not find any other clusters including both diploid and tetraploid, except for a cluster of $\operatorname{clm} 24$ and umb21, which was not supported by a high statistical value.

\section{DISCUSSION}

The primary purpose of this study was to determine the lineage of the $\mathrm{U}$ genome in two UM genome tetraploid species, Ae. columnaris and Ae. triaristata. The PCR products of the U31 region demonstrated amplification length polymorphism for these two species and the Ugenome diploid Ae. umbellulata. The size difference was caused by a 123bp large deletion for both diploid and tetraploid. This result indicated that at least two distinct genetic sources of Ae. umbellulata contributed to the $\mathrm{U}$ genome of the two tetraploids. CAPS analysis with $M s p I$ also revealed two restriction patterns common to the three species. Sequence analysis, however, indicated that the non-cleaved type (allele II) was generated several times by parallel mutations at the $M s p$ I site. Therefore, the CAPS variation does not represent an evolutionary process of the $\mathrm{U}$ genome. Although the outgroup species Ae. bicornis possessed CTGG at the MspI site, the entire sequence of the U31 region was much different from that of any U genome species. The plasticity of the $M s p I$ site implies the possibility of a parallel mutation in Ae. bicornis, so that we could not determine if the CTGG at the $M s p$ I site represents an ancestral sequence of the $\mathrm{U}$ genome.

In the phylogenetic tree, a distinct cluster comprising two Ae. umbellulata accessions and one Ae. columnaris accession (umb28, umb31 and clm07) was formed, which indicates the presence of a third origin of the $U$ genome of Ae. columnaris (Fig. 3). A distinct cluster containing three accessions of Ae. triaristata (trr01, trr02 and trr37) was also found, but this cluster did not include diploid accessions. Although the three accessions of Ae. triaristata had CCTG at the MspI site, three Ae. umbellulata accessions possessing the same nucleotides at the $M s p I$ site did not show a high level of sequence similarity with them (data not shown). Those distinct sequences of Ae. triaristata may have been derived from another Ae. umbellulata not used in this study. The expected maximum frequency of a rare allele to escape in a sample size of 72 at a $95 \%$ confidence interval is 0.041 . Consequently, estimating conservatively, we conclude that the number of origins of the $\mathrm{U}$ genome is at least three for Ae. columnaris, and at least two, possibly more, for Ae. triaristata.

The frequency and level of variation indicated that the longer type of U31 (alleles I and II) was ancestral and was introduced into the tetraploids first via polyploidization, and that the shorter type (allele III) originated later. A question therefore arises; how was the second type introduced into each tetraploid? Three events could have been involved in the process, namely, (i) independent second polyploidization, (ii) introgression from diploid to tetraploid by interploidal hybridization, and (iii) gene flow between tetraploids. It seems possible that any of these might have occurred because evidence for each event has been reported in the genus Aegilops (Murai and Tsunewaki, 1986; van Slageren, 1994; Guadagnuolo et al., 2001). Furthermore, the high level of sequence similarity of allele III among the three species obscures the process responsible for the second origin. The geographical distribution, however, gives a hint. Allele III of Ae. umbellulata was restricted to western Turkey and Greece, whereas that of the two tetraploids was found in eastern Turkey and eastward. The lack of overlap of the distribution rules out recent and frequent introgression from diploid to tetraploid. On the other hand, the distributions of allele III for the two tetraploids overlap with each other in northern Iraq. Moreover, our unpublished AFLP data indicated that the accessions of Ae. triaristata possessing allele III are genetically closer to Ae. columnaris than the other Ae. triaristata. Hence, we deduced that allele III was introduced from diploid to a tetraploid at a considerably early time by a second polyploidization or interploidal introgression, and the other tetraploid gained the allele by gene flow between the tetraploids.

Recent molecular analyses have suggested that the evolutionary features of the formation of polyploids are more complex than previously thought. Regarding the Aegilops-Triticum group, multiple origins of polyploids have been clarified in several cases; Ae. triuncialis (Murai and Tsunewaki, 1986; Chee et al., 1995; Vanichanon et al., 2003), Ae. biuncialis (Chee et al., 1995), T. aestivum L. (Talbert et al., 1998; Caldwell et al., 2004), Ae. cylindrica Host (Caldwell et al., 2004), and Ae. columnaris and Ae. triaristata in this study. These results indicate that multiple origins are the general rule for polyploid species in these genera. Another question is how the multiple species sharing the common UM genome formed via polyploidization. Regarding this question, two proposed hypotheses have remained controversial for a long time. One hypothesis postulates the independent origin, 
namely, each tetraploid species was derived through independent polyploidization (Kihara, 1954; 1963). The other is called the pivotal-differential theory, that is, the speciation of tetraploids was caused by rearrangements of the differential genomes at a tetraploid level (Zohary and Feldman, 1962). In both theories, it is considered that the speciation of tetraploids is caused by variation of the $\mathrm{M}$ genome while the $\mathrm{U}$ genome is stable and less variable. Contrary to these previous hypotheses, our present study indicated that the $\mathrm{U}$ genome is also variable and was introduced to each tetraploid multiple times. Further investigation of the speciation among the polyploid species will be required to elucidate the "origin of species" via polyploidization.

We would like to thank Drs J. Valkoun and K. Chabane, Genetic Resources Unit, ICARDA, for their kind selection of the material of Ae. umbellulata and providing it to us. We are sincerely grateful to Dr. T. Sasakuma for his continuous encouragement and variable comments throughout this study. This study was supported by Research Fellowships of Kihara Memorial Yokohama Foundation for the Advancement of Life Sciences.

\section{REFERENCES}

Badaeva, E. D. (2002) Evaluation of phylogenetic relationships between five polyploid Aegilops L. species of the U-genome cluster by means of chromosome analysis. Genetika 38, 799-811 (in Russian with English summary).

Caldwell, K. S., Dvorak, J., Lagudah E. S., Akhunov, E., Luo, M. C., Wolters, P., and Powell, W. (2004) Sequence polymorphism in polyploid wheat and their D-genome diploid ancestor. Genetics 167, 941-947.

Chee, R. W., Lavin, M., and Talbert, L. E. (1995) Molecular analysis of evolutionary patterns in $\mathrm{U}$ genome wild wheats. Genome 38, 290-297.

Dekaprelevich, L. L. (1961) The species Triticum macha Dek. et Men. In the light of the most recent investigations on the origin of hexaploid wheat. Zeitschr. Pfl. Züchtung 45, 1730 .

Guadagnuolo, R., Savova-Bianchi, D., and Felber, F. (2001) Gene flow from wheat (Triticum aestivum L.) to jointed goatgrass (Aegilops cylindrica Host.), as revealed by RAPD and microsatellite markers. Theor. Appl. Genet. 103, 1-8.

Kihara, H. (1954) Consideration on the evolution and distribution of Aegilops species based on the analyser-method. Cytologia 19, 336-357.

Kihara, H. (1963) Interspecific relationship in Triticum and Aegilops. Seiken Ziho 15, 1-12.

Kihara, H., and Tanaka, M. (1970) Addendum to the classification of the genus Aegilops by means of genome-analysis. Wheat Inform. Serv. 70, 1-2.

Kimber, G., and Feldman, M. (1987) Wild wheats: An introduction. Special Report No. 353, University of Missouri-Colum- bia, Columbia.

Kuckock, V. (1964) Experimental investigation on the origin of wheat. Zeitschr. Pfl. Züchtung 51, 97-140.

Levin, D. A. (1980) Polyploidy and distribution. In: Polyploidy: Biological relevance (ed.: W. H. Lewis), pp. 45-60. Plenum, New York.

Lilienfeld, F. A. (1951) H. Kihara: Genome-analysis in Triticum and Aegilops. X. Concluding review. Cytologia 16, 101123.

MacKey, J. (1966) Species relationships in Triticum. In: Proc. 2nd Intl. Wheat Symp., Lund, Sweden (ed.: J. MacKey), pp. 237-276. Berlingska Boktryckeriet Lund, Lund.

Masterson, J. (1994) Stomatal size in fossil plants: evidence for polyploidy in majority of angiosperms. Science 264, 421424.

Murai, K., and Tsunewaki, K. (1986) Molecular basis of genetic diversity among cytoplasms of Triticum and Aegilops species. IV. CtDNA variation in Ae. triuncialis. Heredity 57, $335-339$.

Murray, M. G., and Thompson, W. F. (1980) Rapid isolation of high molecular weight plant DNA. Nucleic Acids Res. 8, 4321-4325.

Osborn, T. C., Pires, J. C., Birchler, J. A., Auger, D. L., Chen, Z. J., Lee, H. S., Comai, L., Madlung, A., Doerge, R. W., Colot, V., and Martienssen, R. A. (2003) Understanding mechanisms of novel gene expression in polyploids. Trends Genet. 19, 141-147.

Resta, P., Zhang, H. B., Dubcovsky, J., and Dvorak, J. (1996) The origins of the genomes of Triticum biunciale, T. ovatum, T. neglectum, T. columnare, and T. rectum (Poaceae) based on variation in repeated nucleotide sequences. Am. J. Bot. 83, 1556-1565.

Sasanuma, T., Chabane, K., Endo, T. R., and Valkoun, J. (2004) Characterization of genetic variation in and phylogenetic relationships among diploid Aegilops species by AFLP: incongruity of chloroplast and nuclear data. Theor. Appl. Genet. 108, 612-618.

Swofford, D. L. (1993) PAUP (Phylogenetic Analysis Using Parsimony) version 3.1.1. Computer program distributed by the Illinois Natural History Survey, Champaign, Ill.

Talbert, L. E., Smith, L. Y., and Blake, N. K. (1998) More than one origin of hexaploid wheat is indicated by sequence comparison of low-copy DNA. Genome 41, 402-407.

Vanichanon, A., Blake, N. K., Sherman, J. D., and Talbert, L. E. (2003) Multiple origins of allopolyploid Aegilops triuncialis. Theor. Appl. Genet. 106, 804-810.

van Slageren, M. W. (1994) Wild wheats: a monograph of Aegilops L. and Amblyopyrum (Jaub. \& Spach) Eig (Poaceae). Joint publication of ICARDA, Aleppo, Syria and Wageningen Agricultural University, The Netherlands.

Wang, G. Z., Miyashita, N. T., and Tsunewaki, K. (1997) Plasmon analyses of Triticum (Wheat) and Aegilops: RCR-single-strand conformational polymorphism (PCR-SSCP) analyses of organellar DNAs. Proc. Natl. Acad. Sci. USA 94, 14570-14577.

Zohary, D., and Feldman, M. (1962) Hybridization between amphidiploids and the evolution of polyploids in the wheat (Aegilops-Triticum) group. Evolution 16, 44-61. 